TITLE:

\title{
Non-contact imaging of pipe thinning using elastic guided waves generated and detected by lasers
}

\author{
$\operatorname{AUTHOR}(\mathrm{S})$ :
}

Hayashi, Takahiro

\section{CITATION:}

Hayashi, Takahiro. Non-contact imaging of pipe thinning using elastic guided waves generated and detected by lasers. International Journal of Pressure Vessels and Piping 2017, 153: 26-31

ISSUE DATE:

2017-06-01

URL:

http://hdl.handle.net/2433/229082

\section{RIGHT:}

(c) 2017. This manuscript version is made available under the CC-BY-NC-ND 4 . license

http://creativecommons.org/licenses/by-nc-nd/4.0/; The full-text file will be made open to the public on 1 June 2019 in accordance with publisher's 'Terms and Conditions for Self-Archiving':; This is not the published version. Please cite only the published version.; この論文は出版社版でありません。引用の際には出版社版をご確認ご利用ください。 
Non-contact imaging of pipe thinning using elastic guided waves generated and detected by lasers

Takahiro Hayashi

\section{Abstract}

Defect images in a plate can be obtained by the generation and detection of flexural elastic waves using a pulsed laser and a laser Doppler vibrometer. The author has developed an experimental system-called an E-camera-that establishes extremely fast imaging. This paper describes the application of the E-camera to imaging of pipe thinning. The first experiments using a straight pipe revealed that resonance patterns appear significantly at certain frequencies due to guided waves propagating both in the longitudinal and circumferential directions, which is a unique characteristic of pipe structures. A pipe thinning located at various circumferential positions could also be visualized appropriately. Moreover, pipe thinning was successfully detected in both straight and branch pipes located at a distance of $6.0 \mathrm{~m}$ from the E-camera system.

Keywords: Laser ultrasonics; Defect imaging; Pipe inspection, Non-contact NDT

\section{Introduction}

A large number of pipes in industrial plants such as chemical plants and nuclear and thermal power stations are aging, and pipe maintenance is becoming a major burden for plant operators. Although visible pipe surfaces can be inspected easily with human eyes, optical cameras, and/or infrared cameras, invisible inner defects such as pipe thinning due to erosion and corrosion have to be inspected using more laborious techniques such as X-rays and ultrasonic testing [1, 2]. In X-ray testing, the transmission intensity of the $\mathrm{X}$-rays, corresponding to the wall thickness of a pipe, is projected onto a film. Although one can inspect pipe thinning such as erosion and corrosion easily using the X-ray image, it requires installation of large equipment and a film located close to the pipe to be inspected. In ultrasonic testing, pipe thickness is measured with an ultrasonic pulse/echo method using a contact transducer. Although ultrasonic testing can be implemented easily without special care and protection devices like X-rays, one can inspect only a small area under the transducer in a single measurement. In other words, these techniques require much greater time and cost than the visual inspection in the process of installation of equipment, preparation, measurement, and evaluation. Moreover, pipes located at great heights require scaffolding to facilitate the inspection, 
and large-scale work would be necessary even for inspecting small areas.

Therefore, efficient ultrasonic inspection using guided waves propagating in the longitudinal direction in a pipe has attracted much attention [3-6]. Most guided-wave inspection systems employ piezoelectric ultrasonic transducer arrays or magnetostrictive sensors for generating and receiving a torsional mode because the torsional mode is effective for long range inspection owing to its prominent characteristics of no dispersion and small attenuation. However, in the guided wave pipe inspection, one encounters the problem of a trade-off between long-range propagation and defect detectability. Although a low frequency range should be used for long range propagation, guided waves in the high frequency range are necessary for detecting small defects [7, 8]. Moreover, guided wave pipe inspection still has an issue regarding the reliable inspection of complex pipe systems like branch pipes and a pipe elbows because guided waves distort significantly at non-straight areas [9].

The author and his colleagues have previously described a new defect-imaging technique using the characteristics of low frequency flexural guided waves and proved that defect images can be obtained with non-contact measurements using plate specimens engraved artificial defects on the back surface [10-15]. Elastic flexural waves were generated by the thermo-elastic effect in response to the laser irradiation of the surface of a plate, and the elastic waves propagating in the plate were detected by a laser Doppler vibrometer (LDV). Because the energy of the elastic wave generated at the laser spot corresponds to the plate thickness at the point where the laser beam impinges and the defect depth in the vicinity of the laser source, defect images can be created from the amplitude distributions obtained by scanning the laser source. The author and his colleagues have developed a fast imaging system, in which defect images can be created within a few seconds by non-contact measurements simulating an optical camera.

Fundamental studies on the "Elastic wave camera (E-camera)" have previously been conducted for flat plates. The present study investigates defect imaging using the E-camera system for pipes that are in the greatest demand in plant maintenance.

\section{Outline of the E-camera}

In the E-camera, an A0 mode of Lamb waves in a low frequency range is generated by a pulsed laser in a plate-like structure and the flexural vibration is detected with the LDV at a surface of the plate. If the thickness at the laser radiation point is small or if defects (reflection sources) are located in the vicinity of the laser radiation point, the energy of the generated flexural waves varies. For example, large signals are detected 
by the LDV for a plate with a defect on the back surface when the laser pulse for generating elastic waves is incident on the surface opposite to the defect area. On the contrary, when the laser impinges on an intact area, the LDV detects smaller signals as shown in Fig. 1. Using this characteristic of flexural elastic waves, rastering the laser source and mapping the distribution of signal amplitude or frequency spectrum peak detected by a fixed receiving device provide a defect image as shown in the lower sketch of Fig. 1 [10-15]. Because this imaging technique works in the frequency range of audible sound for a plate with the thickness on the order of a few $\mathrm{mm}$, non-contact detection using air-coupled transducers has become feasible [11]. The use of the low frequency range leads to the resonance patterns in the images. Because the resonance patterns differ for different frequencies and receiving positions, the author proved that taking an average of multiple images at different frequencies and positions provides a clearer defect image [11, 13, 15]. In [14], non-contact LDV measurements were implemented with high signal-to-noise ratio (SNR) in the frequency domain at a low peak energy with modulated laser pulse trains generated by a high-repetition-rate fibre laser instead of the Nd:YAG laser with giant pulse energy that is generally used for laser ultrasonic generation. In [15], the effect of high-speed imaging was discussed and it was proved that defect images can be obtained within a few seconds. Moreover, [13] demonstrated that this imaging technique can provide an image in complex plate-like structures where a direct propagation path does not exist. The results obtained so far imply that irradiating a plate-like structure with pulsed laser beams provides defect images within a few seconds. Because one can create defect images easily with a mode of operation that is similar to an optical camera, we call this measurement system an "E-camera".

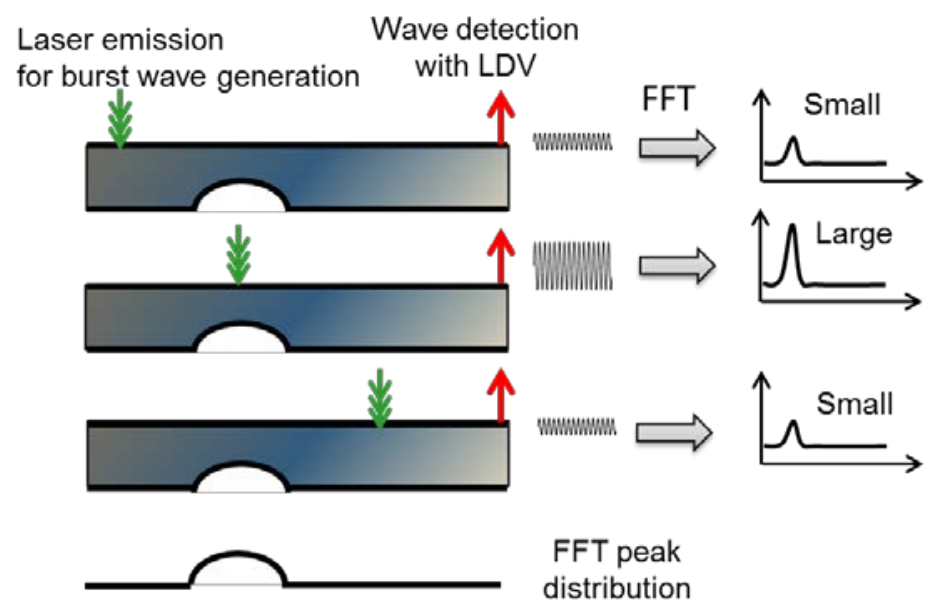

Fig. 1 Principle of defect imaging using the E-camera. 


\section{Imaging a straight pipe}

Fig. 2 shows the E-camera system used in this study. The fibre-laser equipment irradiates the test object with high-repetition-rate laser pulses modulated by external rectangular signals. The modulation frequency of the burst signals was varied from 22 $\mathrm{kHz}$ to $36 \mathrm{kHz}$ as shown later, and the time duration of the burst signals was set to 1500 $\mu$ s. The use of fiber laser equipment and the modulation of the laser pulse emission enable us to generate long-duration burst waves, leading to improve signal to noise ratio in frequency domain $[14,15]$ The elastic wave generated by the fibre laser propagated in the pipe as a guided wave, and was then detected by the LDV (Polytec OFV-5000) at the edge of the pipe. Compared with the experiments of high-speed imaging in [15], in this study, laser pulse trains were generated at the larger interval of $12.5 \mathrm{~ms}$, and waveforms at $10 \mathrm{~ms}$ after the laser emission were used for taking the FFT to maintain a high SNR in the frequency domain. Thus, because the laser source repeatedly moves at $12.5 \mathrm{~ms}$ intervals, the required time was approximately $12.5 \mathrm{~ms} \times$ the number of rastering points. The distances between the E-camera system and the pipes were $2.6 \mathrm{~m}$ and $6.0 \mathrm{~m}$ in experiments. Laser spots were about $3.0 \mathrm{~mm}$ in both cases owing to a collimator lens attached to the fibre laser equipment.

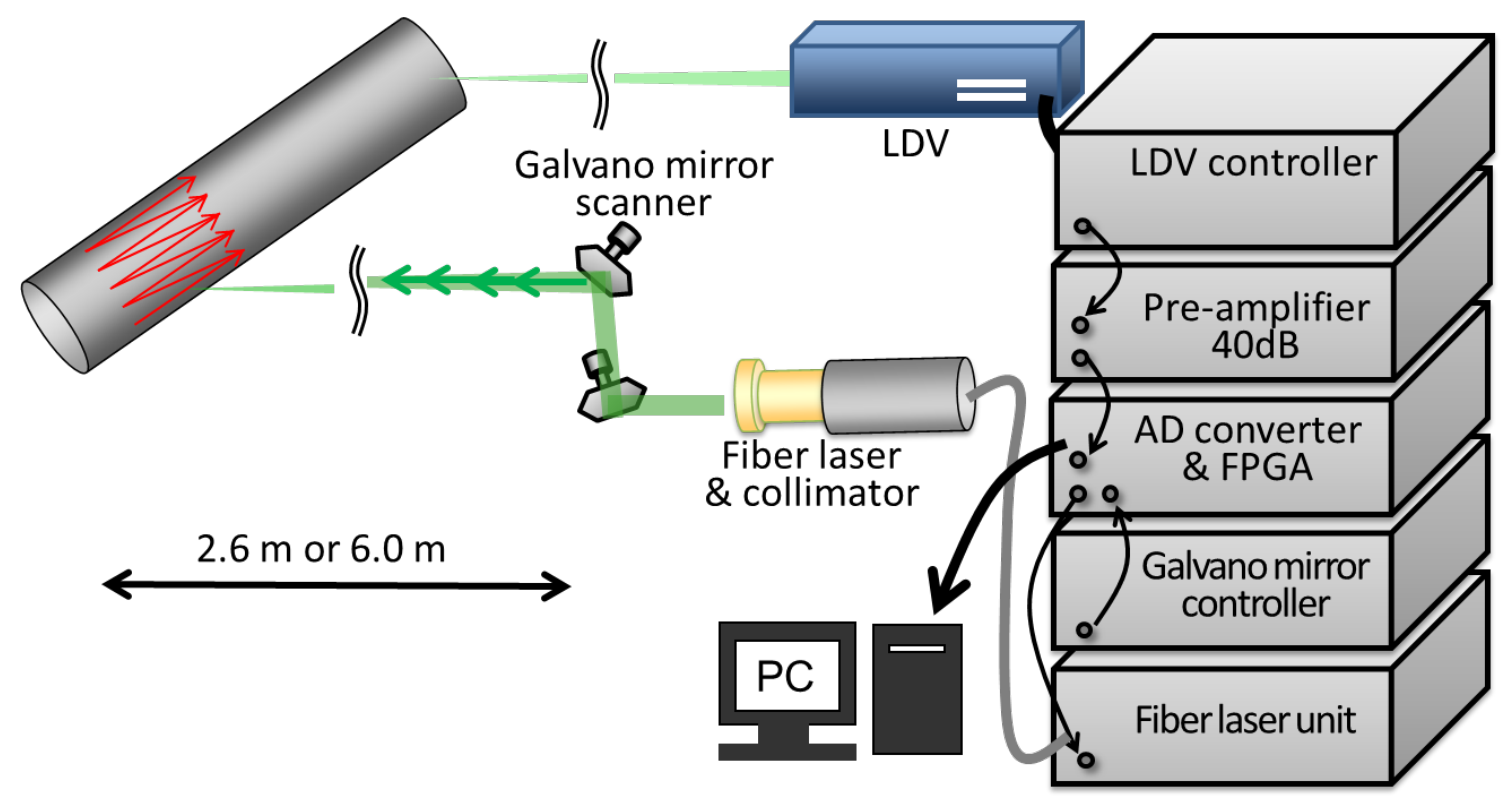

Fig. 2 Schematic figure of the E-camera system.

Fig. 3 shows the straight aluminium alloy (A5052) pipe used in the experiments; it had a length of $1.0 \mathrm{~m}$, an outer diameter of $100 \mathrm{~mm}$, and a thickness of $5.0 \mathrm{~mm}$; an artificial thinning was created with a lathe at $420 \mathrm{~mm}$ from the left edge. The minimum 
thickness of the thinned region is $2.5 \mathrm{~mm}$ and its width is $50 \mathrm{~mm}$. To obtain the complete image of the pipe, the laser source was rastered on the rectangular region measuring $1080 \mathrm{~mm} \times 140 \mathrm{~mm}$ in $2.0 \mathrm{~mm}$ increments. The elastic wave was detected with the LDV at the centre of the right edge. A retro-reflection tape was attached to the receiving area to facilitate stable measurement with the LDV.

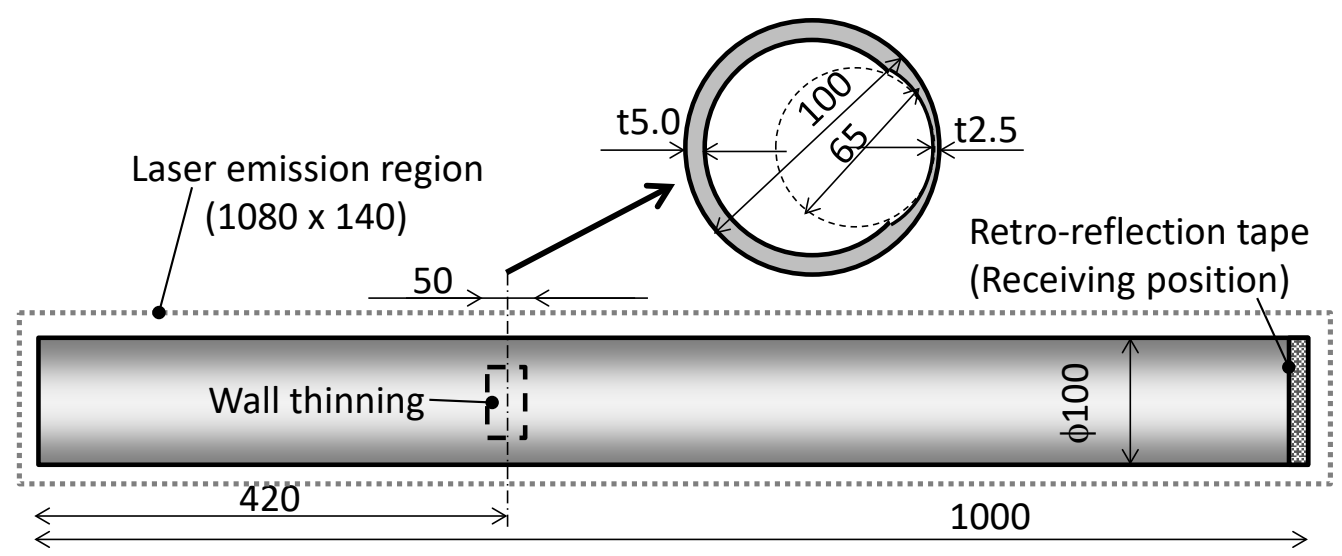

Fig. 3 Straight test pipe used in this study.

First, in order to demonstrate differences of images as a function of frequency, Fig. 4 shows the distributions of a frequency spectrum peak at different modulation frequencies from $22 \mathrm{kHz}$ to $36 \mathrm{kHz}$. The distance between the pipe and the E-camera system was about $2.6 \mathrm{~m}$ and the line of the minimum thickness in the thinned region of the pipe was positioned at the centre of the rastering region. Although resonance patterns varied significantly as a function of frequency, the pipe thinning area was commonly indicated as a darker region. For example, in (b) at $24 \mathrm{kHz}$, longitudinal patterns repeat about every $26 \mathrm{~mm}$ and transverse patterns consist of four nodes in the pipe circumference. In (f) at $32 \mathrm{kHz}$, unique elongated patterns can be seen between the right edge of the pipe and the thinned region of the pipe. 


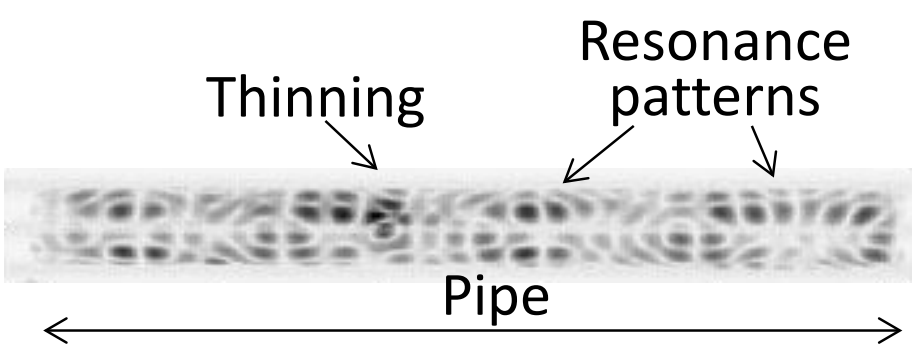

(a) $22 \mathrm{kHz}$

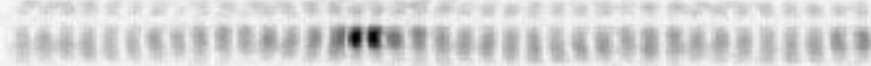

(b) $24 \mathrm{kHz}$

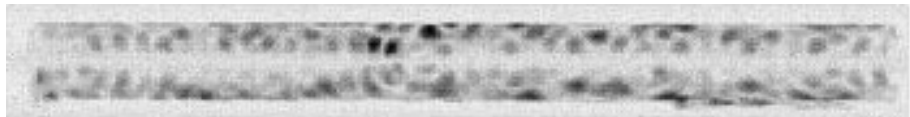

(c) $26 \mathrm{kHz}$

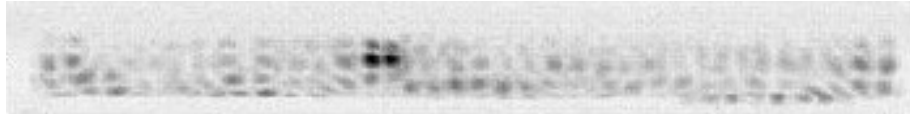

(d) $28 \mathrm{kHz}$

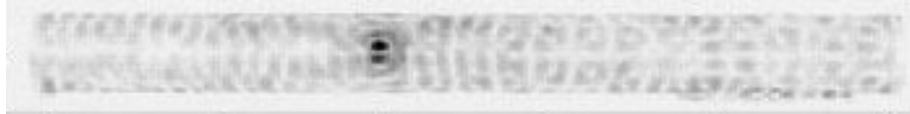

(e) $30 \mathrm{kHz}$

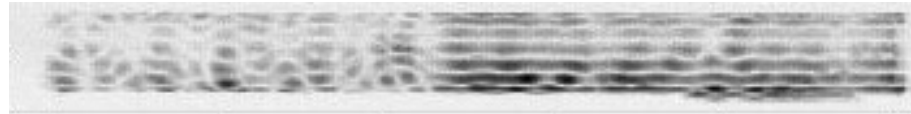

(f) $32 \mathrm{kHz}$

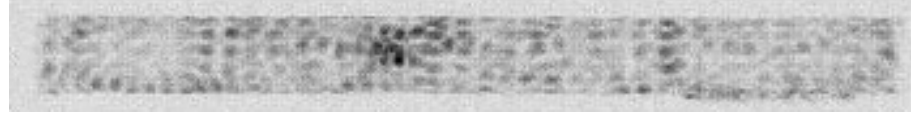

(g) $34 \mathrm{kHz}$

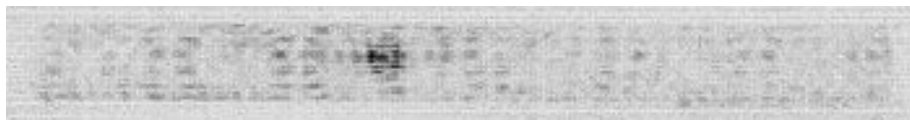

(h) $36 \mathrm{kHz}$

Fig. 4 Differences of images as a function of frequency

For the modal analysis, phase velocity dispersion curves for guided waves propagating in the longitudinal direction in the pipe were calculated as shown in Fig. 5. The calculation was carried out with a semi-analytical finite element method (SAFEM) $[16,17]$, in which the longitudinal and transverse velocities in the aluminium alloy pipe 
were set to $6400 \mathrm{~m} / \mathrm{s}$ and $3100 \mathrm{~m} / \mathrm{s}$, respectively. The point (b) in the dispersion curves indicates a flexural mode with the circumferential order $n=2$ and wavelength $\lambda=52$ mm. Guided wave modes with the circumferential order $n=2$ provide a pattern having 4 nodes in the circumferential direction and $\lambda / 2$ periods in the longitudinal direction [3, 18, 19]. This agrees well with the resonance pattern of Fig. 4 (b). The point (f) in Fig. 5 indicates a mode with $n=8$ and very large phase velocity, which implies that this mode forms a resonance pattern with 16 nodes in the circumferential direction and a very long distribution stretching in the longitudinal direction. This also agrees well with the experimental resonance pattern shown in Fig. 4 (f). In other words, the images obtained with the E-camera as shown in Figs. 4 (b) and (f) were formed mainly by the modes indicated by (b) and (f) in Fig. 5. The other complex patterns in Fig. 4 may be caused by multiple resonance modes.

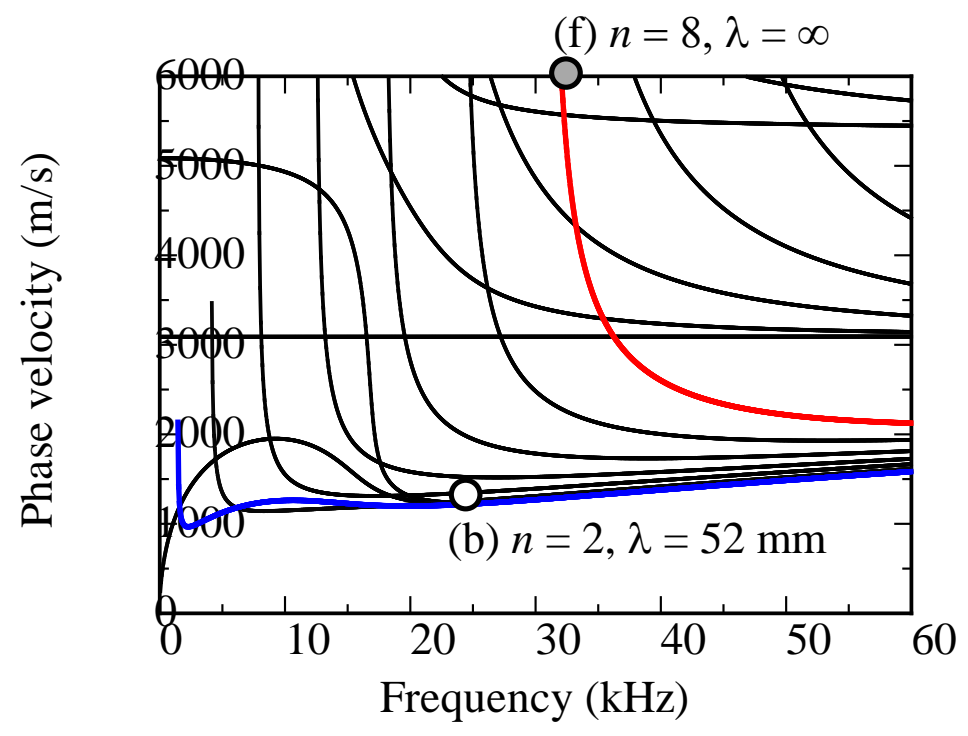

Fig. 5 Phase velocity dispersion curves for the aluminium alloy pipe.

The resonance patterns, which are undesirable for imaging defects, were also observed in flat plates [12-14]. In the previous studies, multiple images obtained at different receiving positions or at different frequencies were averaged to reduce the resonance patterns that strongly depend on receiving position and frequency. In the current experiments, frequency image averaging (FIA) was carried out as described in [15]. The burst modulation waves consisted of three rectangular waves at the frequencies of $26 \mathrm{kHz}, 30 \mathrm{kHz}$, and $34 \mathrm{kHz}$, whose time duration was $1500 \mu$ s each. Three distributions of frequency spectrum peaks were obtained in the three frequency bands, and then the averaged image of the distributions was created as shown in Fig. 6. 
Just as in the case of a flat plate [15], resonance patterns in the case of the pipe were also reduced by FIA, and the area of pipe thinning was enhanced. In real inspection of pipeworks, although the resonance patterns may change depending on the arrangement of pipe supports, weld, and other complex conditions, the FIA will work effectively to reduce resonance patterns and to enhance defect images.

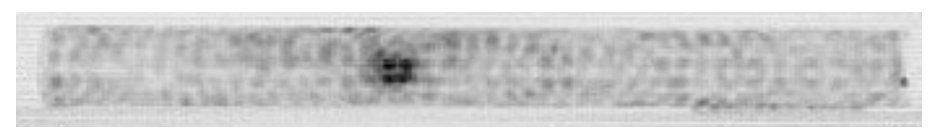

Fig. 6 Averaged image for the modulation frequencies of $26 \mathrm{kHz}, 30 \mathrm{kHz}$, and $34 \mathrm{kHz}$.

Fig. 7 shows the averaged images for a pipe thinning located at different circumferential positions in a pipe in order to investigate the effect of circumferential position on the image. These figures are the averaged images when the pipe used in Fig. 4 and 5 was rotated at (a) $45^{\circ}$ and (b) $90^{\circ}$, and the thinned region of the pipe moved in an upward direction in the image. These figures demonstrate that a dark area indicating the pipe thinning is obtained even when the pipe thinning is not located at the centre of the pipe. In the case of a pipe, the incident angle of the laser beam, laser spot size, and energy density differ depending on the circumferential position. Here, the incident beam is collimated with a diameter of about $3.0 \mathrm{~mm}$, and the laser spot becomes an ellipse whose major axis varies as a function of the circumferential position on the pipe surface. However, energy of the generated elastic wave did not vary significantly with the change of shape of the laser spot because the wavelength used in the experiments was significantly larger than the laser spot size of $3.0 \mathrm{~mm}$. Thus, defect images were appropriately obtained even at $45^{\circ}$ and $90^{\circ}$. The relationship between the incident angle of a laser beam and the generated elastic wave was described in detail in refs. [20, 21], in which it was proved that elastic wave generation is barely affected by the incident angle of the laser beam.

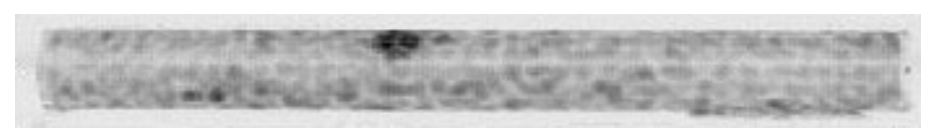

(a) $45^{\circ}$.

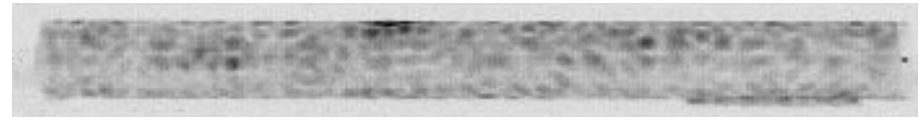

(b) $90^{\circ}$.

Fig. 7 Images of pipe thinning at different circumferential locations. 
In pipe inspection, remote measurements are an important factor for applications in inaccessible areas such as a pipe at a great height and/or at high temperature. Here, to demonstrate the ability of remote measurements with an E-camera, a pipe image was obtained at a distance of $6.0 \mathrm{~m}$ from the pipe (Fig. 8). This figure is an averaged image when the pipe thinning is located at the centre of the circumference as in Fig. 4. The dark area is located at the correct position, which implies that remote measurement from $6.0 \mathrm{~m}$ does not affect the image significantly, although it may cause reductions of detection ability in the LDV and reduced elastic wave generation due to small changes of the laser power density.

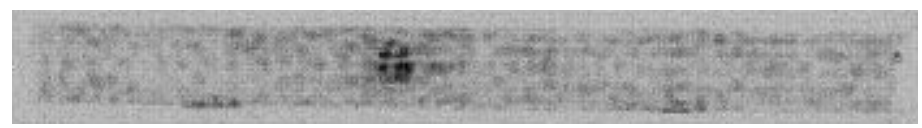

Fig. 8 Defect image in the case of a measurement from a distance of $6.0 \mathrm{~m}$.

\section{Imaging a branch pipe}

As described in [13], this technique can be applied to many complex plate-like structures because an image shows a variation of generation energy caused by the plate thickness and the presence of defects at the laser source. In this section, the imaging technique with the E-camera is applied to a branch pipe as shown in Fig. 9. Two aluminium alloy pipes with a diameter of $100 \mathrm{~mm}$ and a wall thickness of $3.0 \mathrm{~mm}$ were welded as shown in the figure, and an artificial pipe thinning was created on the inner surface of the branch pipe. A laser source was rastered over the region of $560 \mathrm{~mm} \times 390$ $\mathrm{mm}$ with $2.0 \mathrm{~mm}$ increments, and as shown above, three images at three frequency bands $26 \mathrm{kHz}, 30 \mathrm{kHz}$, and $34 \mathrm{kHz}$ were averaged. The elastic wave was detected at three points where retro-reflective tapes were attached, and the images obtained for these receiving points were compared. Fig. 10 (a), (b), and (c) are the images obtained for the upper, left, and right receiving points, respectively. In (a), despite the fact that the elastic wave generated at the pipe thinning travelled through the junction of the branch pipe, a defect image was properly obtained. Weld lines are lighter in the image because of the rough surface and the thicker cross-section. In (a) and (b), showing the results from the upper and left receiving positions, distinct stripe patterns stretching from the pipe edge were seen. These patterns may be caused by a mode resonating in the circumferential direction with a long wavelength in the longitudinal direction as seen in Fig. 4 (f). In Fig. 11, images at three frequency bands before averaging, clearly show the resonant mode. The distinct stripe pattern observed only in (b) at $30 \mathrm{kHz}$ proves that the stripe pattern shown in Fig. 10 (a) was caused by a circumferentially 
resonating mode at $30 \mathrm{kHz}$. Although the stripe patterns appear faintly at the right edge of the pipe in Fig. 10 (c), they are not clear because of the short length of uniform pipe region at the right edge.

In real pipe inspection, as frequencies of the circumferential resonance can be predicted from the theoretical dispersion curves as shown in Fig.5, we can avoid the resonance in the circumferential direction. The other undesirable patterns caused by the other resonances can be reduced by FIA. On the other hand, the unwanted images due to the surface conditions of the pipe like weld lines were inevitable using E-camera. However, we usually can predict the effect of surface conditions from the pipe design or visual observation, and the imaging technique becomes more effective and reliable using a priori information of pipe defects.

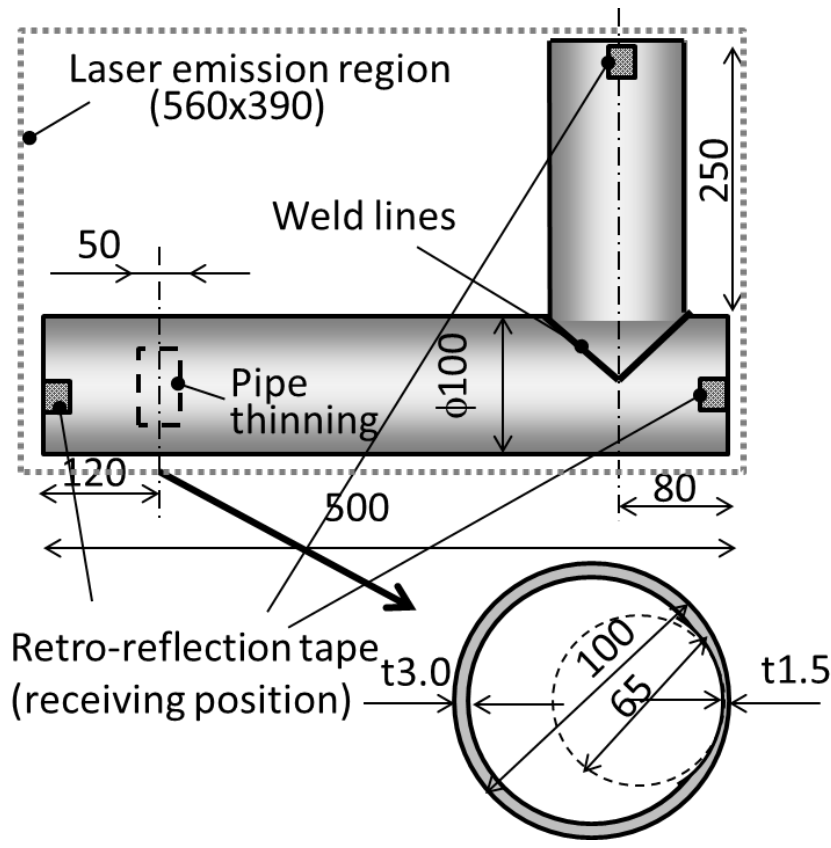

Fig. 9 Branched pipe specimen. 


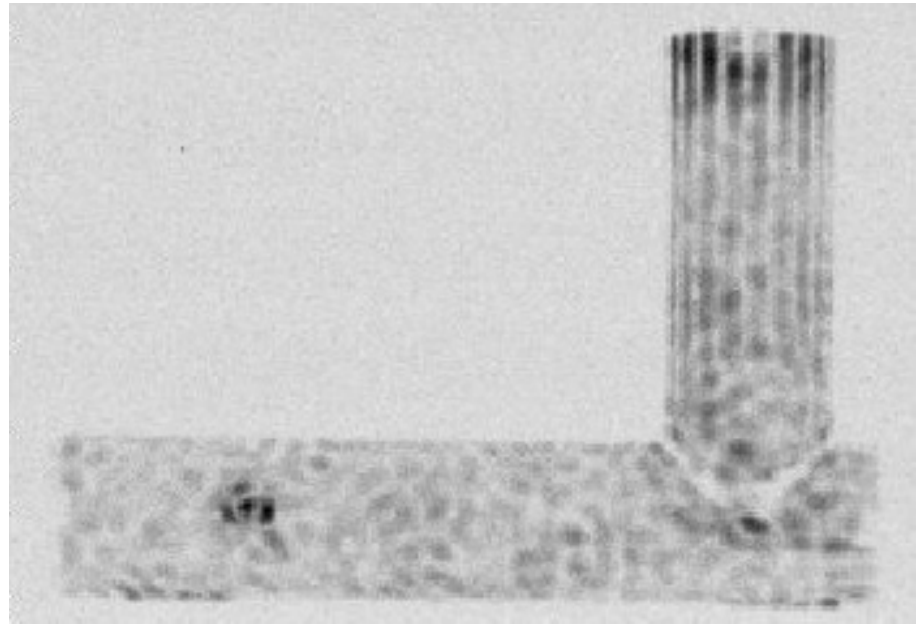

(a) Upper

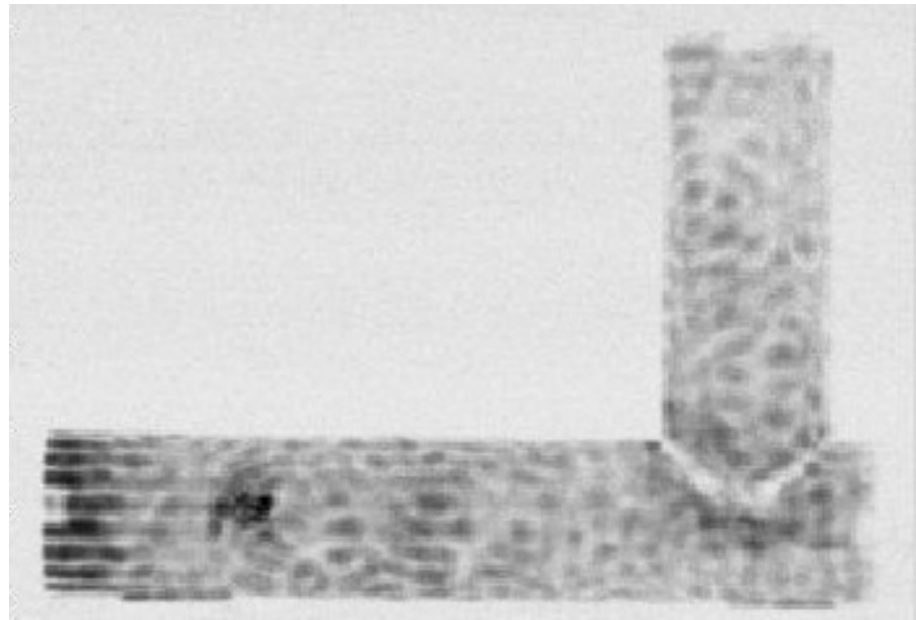

(b) Left

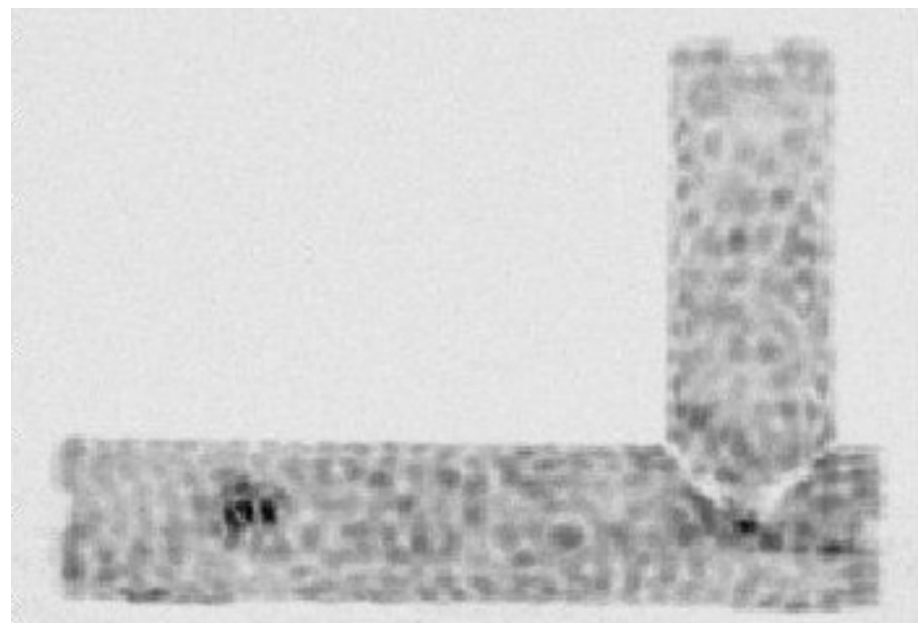

(c) Right

Fig. 10 Images of the pipe branch with frequency image averaging (FIA) for different receiving positions 


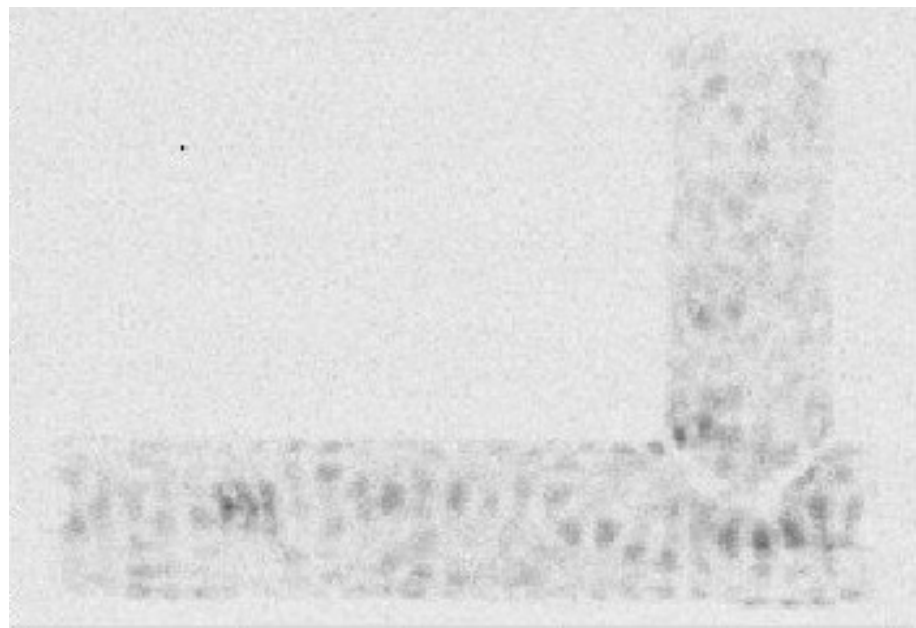

(a) $26 \mathrm{kHz}$

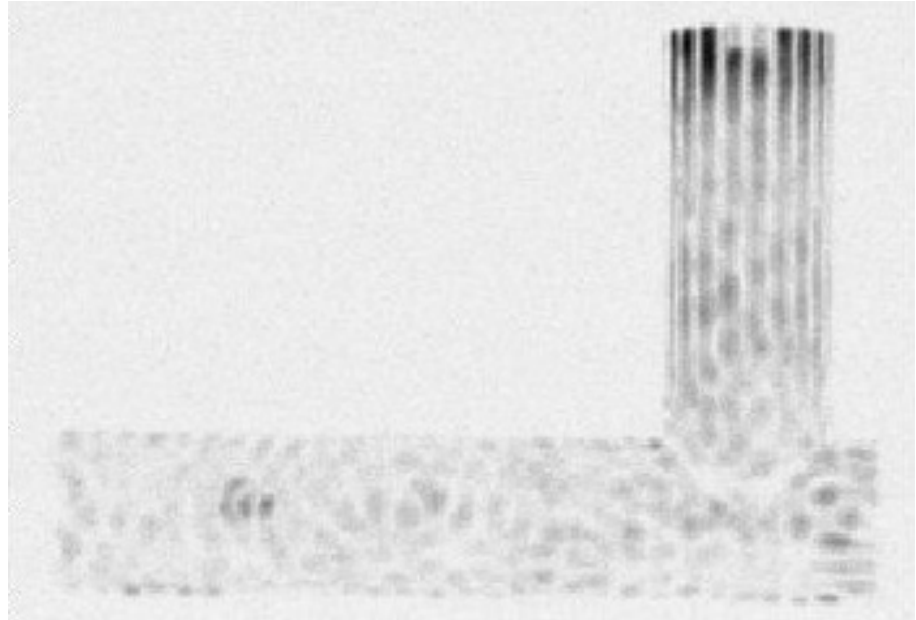

(b) $30 \mathrm{kHz}$

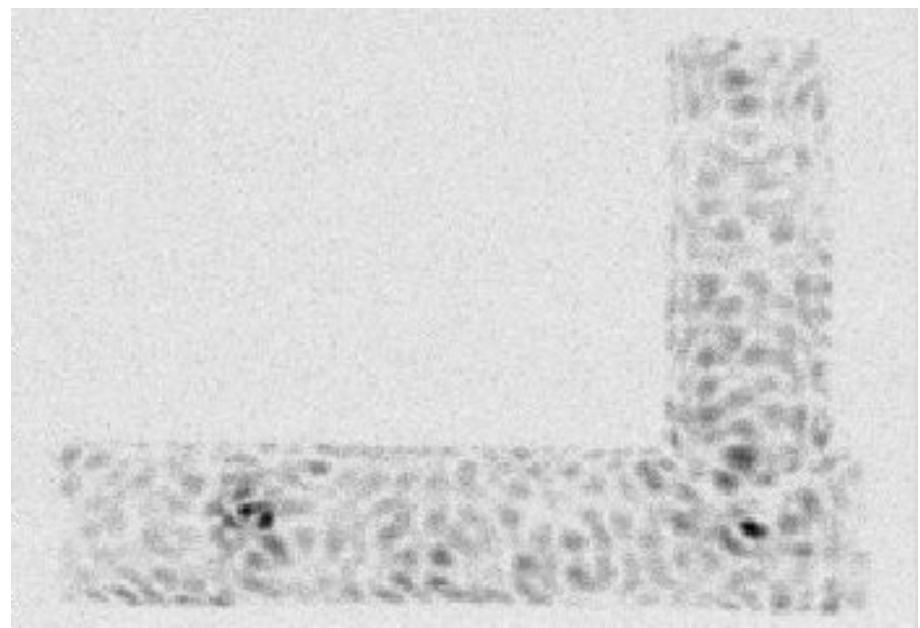

(c) $34 \mathrm{kHz}$

Fig. 11 Frequency spectrum peak distributions for different modulation frequencies.

The receiving position is the upper edge. 


\section{Conclusions}

This study investigated the applicability of defect imaging using an E-camera to the detection of wall thinning in pipes. Artificial thinning in both straight and branched aluminium pipes was visualized properly with the E-camera. Because resonance of guided wave modes propagating in the circumferential direction is possible as well as the resonance in the longitudinal direction, there existed a frequency band in which stripe patterns stretching in the longitudinal direction were observed. Although frequency image averaging is generally effective for reducing spurious resonance patterns, stripe patterns remained in the averaged image when large resonance patterns were included in the selected frequency bands. Although the laser incident angle varies with the circumferential position of the laser spot, an image of pipe thinning was properly obtained for all positions. Moreover, satisfactory images of pipe thinning were obtained from measurements with the E-camera system at distances of both $2.6 \mathrm{~m}$ and $6.0 \mathrm{~m}$ from straight and branched pipes.

Recently, high-power fibre-laser equipment has become widely available for research and development, and LDVs for detecting elastic waves at a long working distance have become practical. Therefore, SNR can be improved even in remote measurements and the imaging technique with the E-camera system is promising for remote non-destructive evaluation of piping systems.

\section{Acknowledgement}

I would like to thank all the staff of workshop in Kyoto University for their effort in the production of pipe specimens. This work was supported by JSPS KAKENHI Grant Number 26282094 and Chubu Electric Power Co., Inc.

[1] M. Palaniappan, R. Subbaratnam, A. Baskaran, R. Chandramohan, The role of non-destructive examination in improving the quality for pipeline and pressure vessels case studies, Int. J. Press. Vessel. Pip. 73 (1997) 33-37.

[2] H.A. Kishawy, H.A. Gabbar, Review of pipeline integrity management practices, Int. J. Press. Vessel. Pip. 87 (2010) 373-380.

[3] J. L. Rose, Ultrasonic Waves in Solid Media (Cambridge University Press, New York, 1999)

[4] P. Cawley, M.J.S. Lowe, D.N. Alleyne, B. Pavlakovic, P. Wilcox, Practical long range guided wave testing: Applications to pipes and rails, Mater. Eval. 61 (2003) 6674.

[5] P.J. Mudge, Field application of the Teletest (R) long-range ultrasonic testing 
technique, Insight. 43 (2001) 74-77.

[6] J.L. Rose, A Baseline and Vision of Ultrasonic Guided Wave Inspection Potential, J. Press. Vessel Technol. 124 (2002) 273.

[7] T. Hayashi, M. Murase, Defect imaging with guided waves in a pipe, J. Acoust. Soc. Am. 117 (2005) 2134-2140.

[8] T. Hayashi, M. Nagao, M. Murase, Defect imaging with guided waves in a long pipe, J. Solid Mech. Mater. Eng. 2 (2008) 888-899.

[9] T. Hayashi, J.L. Rose, Guided wave simulation and visualization by a semianaltytical finite element method, Mater. Eval. 61 (2002) 75-79.

[10] T. Hayashi, M. Murase, M.N. Salim, Rapid thickness measurements using guided waves from a scanning laser source, J. Acoust. Soc. Am. 126 (2009) 1101-1106.

[11] T. Hayashi, M. Murase, T. Kitayama, Frequency dependence of images in scanning laser source technique for a plate, Ultrasonics. 52 (2012) 636-642.

[12] T. Hayashi, M. Murase, N. Ogura, T. Kitayama, Imaging Defects in a Plate with Full Non-Contact Scanning Laser Source Technique, Mater. Trans. 55 (2014) 1045-1050.

[13] T. Hayashi, Imaging defects in a plate with complex geometries, Appl. Phys. Lett. 108 (2016) 081901.

[14] T. Hayashi, K. Ishihara, Generation of narrowband elastic waves with a fiber laser and its application to the imaging of defects in a plate, Ultrasonics. 77 (2017) 4753.

[15] T. Hayashi, High-speed non-contact defect imaging for a plate-like structure, NDT E Int. 85 (2017) 53-62.

[16] T. Hayashi, K. Kawashima, Z. Sun, J.L. Rose, Analysis of flexural mode focusing by a semianalytical finite element method., J. Acoust. Soc. Am. 113 (2003) 1241-1248.

[17] T. Hayashi, W.-J. Song, J.L. Rose, Guided wave dispersion curves for a bar with an arbitrary cross-section, a rod and rail example, Ultrasonics. 41 (2003) 175-183.

[18] D.C. Gazis, Three-dimensional investigation of the propagation of waves in hollow circular cylinders. I. Analytical foundation, J. Acoust. Soc. Am. 31 (1959) 568573.

[19] T. Hayashi, M. Murase, Mode extraction technique for guided waves in a pipe, Nondestruct. Test. Eval. 20 (2005) 63-75.

[20] C.B. Scruby and L.E. Drain, Laser Ultrasonics: Techniques and Applications (Adam Hilger, Bristol, Philadelphia, and New York, 1990).

[21] A. Blouin, D. Levesque, C. Neron, D. Drolet, J.P. Monchalin, Improved 
resolution and signal-to-noise ratio in laser-ultrasonics by SAFT processing., Opt. Express. 2 (1998) 531-539. 